\section{PO-1040 WITHDRAWN}

\section{PO-1041 FAVOURABLE CLINICAL COURSE OF JAPANESE SIBLINGS WITH INTERLEUKIN-1 RECEPTOR-ASSOCIATED KINASE 4 DEFICIENCY}

${ }^{1} \mathrm{H}$ Yoshikawa, ${ }^{2} \mathrm{~S}$ Watanabe, ${ }^{2} \mathrm{~N}$ Togashi. 'Pediatrics, Nagaoka Habilitation and Medical Center, Nagaoka, Japan; ${ }^{2}$ Neurology, Miyagi Children's Hospital, Sendai, Japan

\subsection{6/archdischild-2014-307384.1655}

Background and aims Interleukin-1 receptor-associated kinase 4 (IRAK4) deficiency is a recently recognised immunodeficiency disorder that affects innate immunity through Toll-like receptors. Although $<100$ patients have been reported, the clinical features are not fully clarified. We report here additional clinical information involving two Japanese siblings with IRAK 4 deficiency, who were successfully prevented by prophylactic treatment in the first few years of life (J Pediatr 2010;156:168).

Case report Case 1: A 9-year-old boy was diagnosed with IRAK4 deficiency, as characterised by an IRAK4 gene mutation. The boy received prophylactic treatment, as previously reported. He did not develop severe infections or pyrexia until 5 years of age; however, he subsequently had several episodes of curable bacterial infections without elevation of C-reactive protein (CRP). At present, he had developed normally and antibody production after the usual vaccinations has been normal. Case 2: A 7 -year-old girl, the younger sister of case 1, was diagnosed with IRAK4 deficiency, as characterised by the same mutation and she received the same prophylactic treatment. She did not have pyrexia or severe infections until 4 years of age. She subsequently had several pyrexic episodes without elevation of CRP. The response to vaccinations was normal with the exception of rubella.

Conclusion The clinical course was favourable for both cases. Although they experienced some bacterial infections and pyrexia, the CRP were not elevated, which may reflect the basic mechanism of this disorder. Antibody production after vaccination was normal in both patients.

\section{PO-1041a A RETROSPECTIVE ANALYSIS OF THE 21134 CASES CHILDREN ALLERGENS SCREENING TESTING AND ITS CLINICAL SIGNIFICANCE}

Q Miao, L Xiang. Allergy Department, Beijing Childrens' Hospital, Beijing, China

\subsection{6/archdischild-2014-307384.1656}

Objective To analyse the sensitisation status to common inhalant allergens of suspected allergic children in Beijing Childrens' Hospital in the year of 2013.

Methods Between January 2013 and January 2014, there are a total of 21134 cases conducted the allergen screening testing (Medswiss AllergyScreen allergen testing system, Germany). According to the department at first visit of outpatient children and the major clinical diagnostic information of the inpatient children, the out-patient children or the in-patient children could be further divied into subgroups accordingly. A comparison in the differences of allergen sensitisation pattern between ward patients and outpatients was performed.

Results In the out-patient subjects, the positive rate of sIgE was $45.99 \%$ (8266/17974 cases), the top three positive rate of inhalant allergen were mould (18.57\%), dog hair (16.54\%) and dust mites (15.69\%). However, in the outpatients, the positive rate of sIgE was $29.34 \%$ (927/3160 cases)and the top three positive detection rate were dust mites $(10.95 \%)$, dog hair $(8.48 \%)$, and the mould $(7.95 \%)$ in turn. It was also found that the positive ratio of children suffering from the nose or eye disease (from ear-nose-throat, head and neck surgery and ophthalmology outpatient: 2092/3126 cases, 66.92\%; from ear-nose-throat of head and neck surgery and ophthalmology ward: 14/26 cases, $53.85 \%)$ were significantly higher than the others. Besides, it was considered that the Henoch-Schonlein purpura (HSP)is one kind of disease that closely related with the sensitisation condition.

In this study, the positive rate to inhaled allergens in HSP childrens was significantly higher than that in non-HSP childrens (HSP: 520/1375 cases, 37.80\%; non-HSP: 407/1782 cases, $22.80 \%, \mathrm{p}<0.0001)$.

Conclusion The allergen screening testing is not only useful in the rapid assessment of body sensitisation state, but also play a positive role in the providing rational allergen avoidance advise. Therefore, it should be to pay more attention in the combination and translation between the allergen screening results and the clinical information.

\section{PO-1041b A COMPARATIVE STUDY OF TWO MEDICATION GROUPS OF INFANTS WITH MODERATE BRONCHIOLITIS}

F Yasin, Z Afridi, R Khan. Paediatrics, Kerry General Hospital, Tralee, Ireland

\subsection{6/archdischild-2014-307384.1657}

Aim To compare effect of Racemic Epinephrine (RE) and 3\% Hypertonic saline (HS) nebulization on length of hospital stay (LOS) of infants with moderate bronchiolitis.

Method Infants with 'moderate' bronchiolitis presenting to Emergency Department (ED) were enrolled (October 2013 through March 2014) as per defined inclusion, exclusion criteria and severity assessment tool. ${ }^{1}$ Ethical approval was obtained. The enrolled infants were assigned RE or HS groups on alternate basis. All infants were monitored minimum every $4 \mathrm{~h}$. Data was entered on Microsoft Excel and SPSS softwares to compare results.

Results Eighty infants with bronchiolitis presented to ED during study period. As per study criteria, 16 infants in HS group and 18 infants in RE group were enrolled. The demographics of the two groups were similar. During the study, one infant from RE group and three infants from HS group were excluded due to progression to severe disease.

Primary outcome In RE group, out of 17 remaining infants, the length of stay ranged between 18-160 h (Mean-45 $\pm 338 \mathrm{~h}$ ). In HS group, out of remaining 13 infants, the length of stay ranged between 18.50-206 h (Mean-83 $\pm 556 \mathrm{~h}$ ).

The mean 'length of stay' in group with Racemic epinephrine alone was significantly reduced (t-test, $\mathrm{p}-0.03$ ) as compared to the Hypertonic saline group.

Secondary outcome No infant in RE group whereas 4 infants in HS group required oxygen therapy. One patient in RE group whereas 2 patients in HS required the intravenous fluids.

Conclusion Racemic Epinephrine nebulization as first-line medication reduces length of hospital stay in infants with moderate bronchiolitis.

\section{REFERENCE}

1. http://www.rch.org.au/dlinicalguide/guideline_index/Bronchiolitis_Guideline/ 